\title{
Swimming exercise inhibits myocardial ER stress in the hearts of aged mice by enhancing cGMP-PKG signaling
}

\author{
PAN CHANG $^{1,2^{*}}$, XIAOMENG ZHANG ${ }^{1,2^{*}}$, MINGYANG ZHANG $^{3 *}$, GUOHUA LI $^{1}$, LANG HU $^{1}$, \\ HUISHOU ZHAO $^{1}$, XIAOXING ZHU ${ }^{1}$, JUAN WU ${ }^{2}$, XIHUI WANG ${ }^{2}$, KAIYAN WANG ${ }^{2}$, JING ZHANG ${ }^{2}$, \\ MINGGANG REN ${ }^{2}$, BAOYING $\mathrm{CHEN}^{4}$, XIAOLING ZHU ${ }^{1}$, MIAOZHANG ZHU ${ }^{1}$ and JUN YU ${ }^{1,4}$ \\ ${ }^{1}$ Department of Physiology, The Fourth Military Medical University, Xi'an, Shaanxi 710032; \\ ${ }^{2}$ Department of Cardiology, The Second Affiliated Hospital, Xi'an Medical University, Xi'an, Shaanxi 710038; \\ ${ }^{3}$ Institute of Forensic Sciences, Soochow University, Suzhou, Jiangsu 215123; \\ ${ }^{4}$ Central Laboratory, Xi'an International Medical Center Hospital, Xi'an, Shaanxi 710100, P.R. China
}

Received February 14, 2019; Accepted June 28, 2019

DOI: $10.3892 / \mathrm{mmr} .2019 .10864$

\begin{abstract}
The purpose of the present study was to explore aging-associated cardiac dysfunction and the possible mechanism by which swimming exercise modulates cardiac dysfunction in aged mice. Aged mice were divided into two groups: i) Aged mice; and ii) aged mice subjected to swimming exercises. Another cohort of 4-month-old male mice served as the control group. Cardiac structure and function in mice were analyzed using hematoxylin and eosin staining, and echocardiography. The levels of oxidative stress were determined by measuring the levels of superoxide dismutase, malondialdehyde and reactive oxygen species (ROS). Levels of the endoplasmic reticulum (ER) stress-related protein PKR-like ER kinase, glucose-regulated protein 78 and C/EBP homologous protein were determined to evaluate the level of ER stress. The aged group exhibited an abnormal cardiac structure and decreased cardiac function, both of which were ameliorated by swimming exercise. The hearts of the aged mice exhibited pronounced oxidative and ER stress, which were ameliorated by exercise, and was accompanied by the reactivation of myocardial cGMP and suppression of cGMP-specific phosphodiesterase type 5 (PDE5). The inhibition of PDE5 attenuated age-induced cardiac dysfunction, blocked ROS production and suppressed ER stress. An ER stress inducer abolished the beneficial effects
\end{abstract}

Correspondence to: Dr Jun Yu or Dr Miaozhang Zhu, Department of Physiology, The Fourth Military Medical University, 169 Changle Road, Xi'an, Shaanxi 710032, P.R. China

E-mail:pclamper@163.com

E-mail: mz_zhu@fmmu.edu.cn

*Contributed equally

Abbreviations: ER, endoplasmic reticulum; TM, tunicamycin; ROS, reactive oxygen species

Key words: aged, ER stress, cardiac function, cGMP, exercise of the swimming exercise on cardiac function and increased ROS production. The present study suggested that exercise restored cardiac function in mice with age-induced cardiac dysfunction by inhibiting oxidative stress and ER stress, and increasing cGMP-protein kinase G signaling.

\section{Introduction}

Aging is an important risk factor for the development of cardiovascular disease (1), and this association may be caused by a continuum of cardiac structural and functional alterations that occur with age (2). These cardiac changes are relevant in age-associated diseases (3). Since aging increases the risk of cardiac disease and reduces organ function, studies that aim to develop interventions that combat cardiac aging and elucidate the disease mechanisms have significant preclinical and clinical implications. Swimming exercise has recently been shown to improve structural abnormalities and upregulate the antioxidant defense capacity of senescent female rat hearts (4). Oxidative stress, due to excessive reactive oxygen species (ROS) production and impaired antioxidant defense mechanisms, induces a range of pathologies that are believed to be important contributors to the cardiovascular aging process (2,5). Recently, Belaya et al (6) reported that long-term wheel running can protect against age-related cellular stress. The endoplasmic reticulum (ER) is a specialized organelle where the folding and post-translational maturation of almost all membrane proteins, and most secreted proteins, occur (7). Although exercise significantly improves cardiorespiratory fitness, little is known about the impact of physical activity on myocardial function. Many of the pathological changes associated with aging have been attributed to oxidative stresses (8). It has been proposed that endurance exercise training is associated with altered ER function (9). The unfolded protein response (UPR) is a crucial process in maintaining ER homeostasis or inducing cell death in chronically damaged cells; the UPR causes ER stress. ER stress is initiated by the activation of at least three types of stress sensors: i) Inositol-requiring enzyme-1; ii) activating transcription factor 6; and iii) PKR-like ER kinase (PERK) (7). Additionally, a previous report demonstrated that levels of 
the ER chaperones glucose-regulated protein 78 (GRP78) are decreased, whereas levels of the pro-apoptotic mediator C/EBP homologous protein (CHOP) are increased in aged brains $(10,11)$. These previous findings suggested that the ability to maintain ER homeostasis may be disrupted during aging; however, the functional significance of these processes in aged hearts remains unclear. Both oxidative stress and ER stress are involved in physiological and pathophysiological processes associated with aging. Therefore, strategies designed to reduce the aberrant activation of oxidative stress and ER stress in the aged heart are of great interest.

cGMP is a ubiquitous second messenger involved in many cardiovascular processes and is produced by guanylate cyclases (12). The biological activity of cGMP is regulated by cGMP-specific phosphodiesterase type 5 (PDE5) through hydrolytic degradation (13). Previous studies have indicated that protein kinase $\mathrm{G}(\mathrm{PKG})$ activation by cGMP has a role in cGMP-induced myocardial functions (13-15). It has also been reported that PKG activation decreases with aging (15). However, the actions of cGMP-PKG signaling in the aged heart are not fully understood. Therefore, the present study was designed with two aims: i) To determine whether exercise training improves myocardial function via the cGMP-PKG pathway; and ii) to examine whether the endogenous cGMP-PKG system attenuated aged-induced myocardial ER stress.

\section{Materials and methods}

Animals and treatment. A total of 64 male C57Bl/6J mice were obtained from the animal center of the Fourth Military Medical University. All animal experimental procedures and protocols were approved by the Ethics Committee of The Fourth Military Medical University. The animals were studied at 4 (young) and 20 (aged) months of age (ranging approximately $25-40 \mathrm{~g}$ ). They were housed under a 12-h light/dark cycle in temperature $\left(22 \pm 2^{\circ} \mathrm{C}\right)$ and humidity $(55 \pm 10 \%)$-controlled rooms with free access to food and water. The mice were assigned to three groups: i) Young ( $\mathrm{n}=16)$; ii) aged $(\mathrm{n}=24)$; and iii) aged + exercise $(n=24)$. The animals in the exercise group performed swimming exercise, free of any loading, 5 days/week for 8 weeks in water maintained at $32-35^{\circ} \mathrm{C}$. The mice swam for $15 \mathrm{~min}$ on the first day, with the swimming duration increased gradually over a 1 week period to 60 min continuously every day on one protocol. All exercise sessions were performed between 8:00 and 11:00 a.m., as previously described $(10,14)$. The aged mice were intraperitoneally injected with sildenafil ( $3 \mathrm{mg} / \mathrm{kg} /$ day for 3 weeks) or tunicamycin (TM; $2 \mathrm{mg} / \mathrm{kg} /$ day for 2 days) $(13,16)$. Sildenafil and TM were purchased from Sigma-Aldrich; Merck KGaA. The compounds were dissolved in $0.9 \%$ saline for injection. All mice were anaesthetized by inhaling oxygen with $5 \%$ isoflurane at the rate of $11 / \mathrm{min}$ after $24 \mathrm{~h}$ of the last drug administration. The mice were confirmed to be deeply anesthetized after they were immobile for $1 \mathrm{~min}$. To euthanatize the mice, a $25 \%$ volume of $\mathrm{CO}_{2}$ gas was allowed to constantly flow of $0.2 \mathrm{l} / \mathrm{min}$ into the chamber until the lack of respiration and heart beat were detected. The heart tissue was isolated post-mortem.

Echocardiography. Echocardiographic examinations were performed using an ACUSON Sequoia 512 ultrasound machine
(Siemens AG) using 2.5\% isoflurane as an inhalant anesthetic. The measurements were performed using M-mode tracings from the papillary muscles and the apical four-chamber view.

Pathological examination. The excised heart samples were fixed with $4 \%$ paraformaldehyde at $4^{\circ} \mathrm{C}$ for $24 \mathrm{~h}$ and incubated in 80, 90 and 100\% ethanol. Each incubation was performed for $30 \mathrm{~min}$. Subsequently, samples were incubated in $100 \%$ ethanol for $15 \mathrm{~min}$ at room temperature, and were subsequently embedded in paraffin wax. The samples were longitudinally sectioned to a thickness of $5 \mu \mathrm{m}$. The sections were stained with hematoxylin and eosin (Sigma-Aldrich: Merck KGaA) and assessed using an optical microscope (Olympus Corporation; magnification, 40-400x). Sections were incubated with haematoxylin $(0.2 \%)$ for $5 \mathrm{~min}$ at room temperature and with eosin (1\%) for 2 min temperature.

Measurements of the level of superoxide dismutase (SOD) activity and malondialdehyde (MDA) content. The hearts were homogenized with saline and the activity of the antioxidant enzyme SOD and the concentration of MDA were determined using commercially available kits (cat. nos. A001 and A003 respectively; Nanjing Jiancheng Bioengineering Institute), according to the manufacturer's instructions.

Detection of ROS. A reactive oxygen species (ROS) assay kit (cat. no. E004, Nanjing Jiancheng Bioengineering Institute) was used to determine the level of ROS generation. The myocardial tissue was trypsinized with trypsin $(0.4 \%)$ at $37^{\circ} \mathrm{C}$ for $3 \mathrm{~min}$, and then washed with PBS and stained with $10 \mu \mathrm{M}$ $2^{\prime}, 7^{\prime}$-dichlorodihydrofluorescein diacetate for $30 \mathrm{~min}$ at $37^{\circ} \mathrm{C}$. After centrifugation at $1,000 \mathrm{x}$ g for $5 \mathrm{~min}$ at room temperature, the tissues were washed three times with PBS and the fluorescence intensity was analyzed using a fluorescence microplate reader (Molecular Devices, LLC) at an excitation wavelength of $480 \mathrm{~nm}$ and an emission wavelength of $530 \mathrm{~nm}$.

cGMP measurement. The cGMP content in the myocardium was measured using the cGMP complete ELISA kit (cat. no. ADI900164, Assay Designs, Inc.), according to the manufacturer's instructions and as previously described (14). The results were reported as $\mathrm{pmol} \mathrm{cGMP} / \mathrm{mg}$ tissue.

Western blot analysis. Western blot analysis was performed as described in our previous study (17). Briefly, frozen animal heart tissues were homogenized in ice-cold RIPA lysis buffer [500 mM Tris- $\mathrm{HCl}$ (pH 8.0), $150 \mathrm{mM} \mathrm{NaCl}, 1 \%$ Nonidet P-40, $0.02 \%$ sodium azide, $0.1 \%$ SDS, $100 \mu \mathrm{g} / \mathrm{ml}$ PMSF, $1 \mu \mathrm{g} / \mathrm{ml}$ aprotinin and $0.5 \%$ sodium deoxycholate]. The cell extracts were centrifuged for $30 \mathrm{~min}$ at $15,000 \mathrm{x} \mathrm{g}$ at $4^{\circ} \mathrm{C}$ and the supernatants were collected. Protein lysates $(35 \mu \mathrm{g} / \mathrm{lane})$ were separated by $10 \%$ SDS-PAGE and transferred onto PVDF membranes. Membranes were blocked with 5\% non-fat milk for $1 \mathrm{~h}$ at room temperature, and then incubated with the following primary antibodies at $4^{\circ} \mathrm{C}$ overnight: GRP78 (cat. no. 3177, Cell Signaling Technology, Inc.), CHOP (cat. no. 5554, Cell Signaling Technology, Inc.), PKG (cat. no. 3248, Cell Signaling Technology, Inc.), PDE5 (cat. no. SAB2500767, Sigma-Aldrich; Merck KGaA) and $\beta$-actin (cat. no. 3700, Cell Signaling Technology, Inc.). Membranes were then 
A

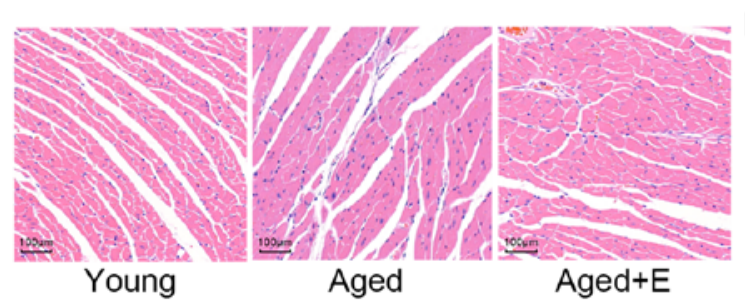

C

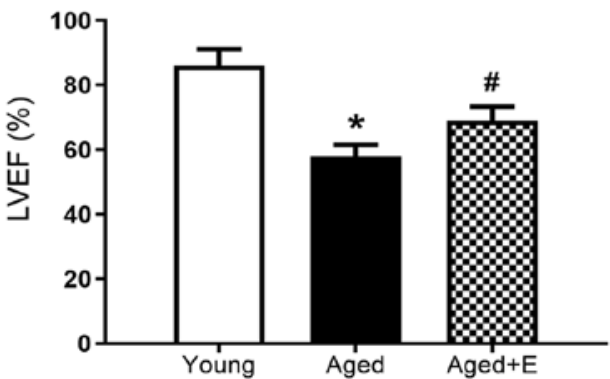

E

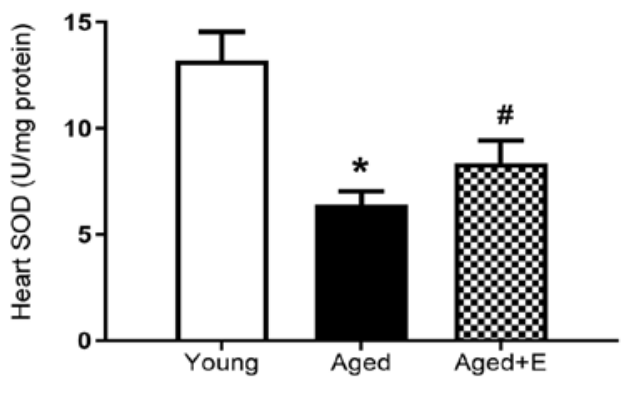

$\mathrm{F}$

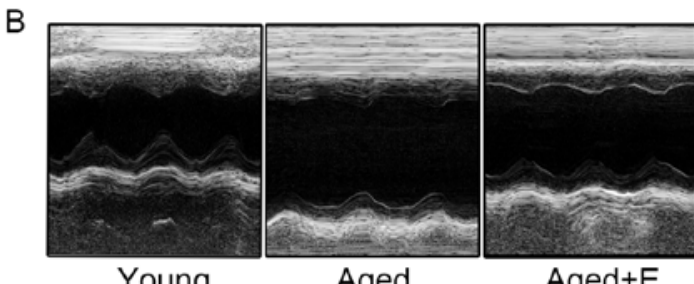

Young
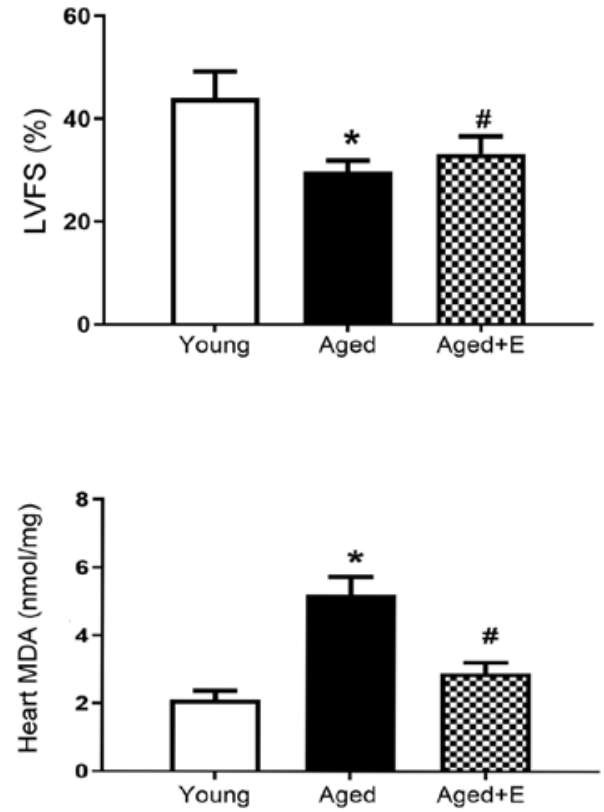

G

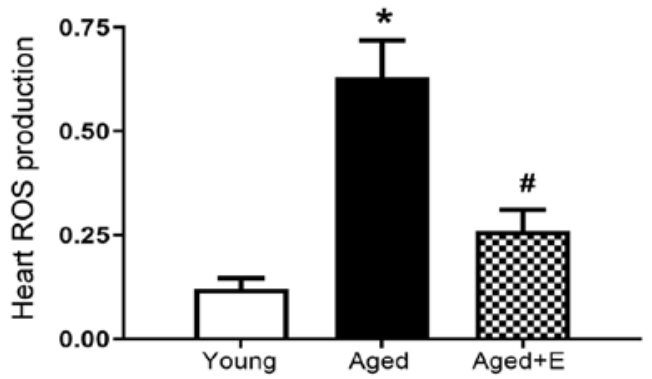

Figure 1. Swimming exercise protects cardiac structure and function. (A) Representative images of hematoxylin and eosin staining of hearts from mice in each group. Scale bar, $100 \mu \mathrm{m}$. (B) Echocardiography of hearts from mice in each group. (C) LVEF and (D) LVFS of hearts from mice in each group. (E) Cardiac SOD activity. $\mathrm{n}=9$. (F) Cardiac MDA level $\mathrm{n}=9$. (G) Cardiac ROS production $\mathrm{n}=9$. Data are presented as the mean $\pm \mathrm{SEM}$. ${ }^{*} \mathrm{P}<0.05$ vs. young mice; ${ }^{\#} \mathrm{P}<0.05$ vs aged mice. LVEF, left ventricle ejection fraction; LVFS, left ventricle fractional shortening; SOD, superoxide dismutase; E, exercise; MDA, malondialdehyde; ROS, reactive oxygen species.

incubated with a peroxidase-conjugated secondary antibody (goat-anti-rabbit; 1:5,000; cat. no. A0208; goat-anti-mouse; 1:5,000; cat. no. A0216; Beyotime Institute of Biotechnology) for 2-3 $\mathrm{h}$ at room temperature before visualization using an Immobilon western ECL hydrogen peroxidase substrate (EMD Millipore; cat. no. WBKLS0500) and analyzed using the Discovery Series Quantity One software (version, 4.52; Bio-Rad Laboratories, Inc.).

Statistical analysis. The results are presented as the mean \pm SEM. Statistical analyses were performed using Student's t-test for comparing two groups or two-way ANOVA for comparing $\geq 3$ groups followed by Bonferroni's post hoc test. All statistical analyses were performed using GraphPad
Prism software version 5.0 (GraphPad Software, Inc.). $\mathrm{P}<0.05$ was considered to indicate a statistically significant difference. All the experiments were repeated for three times.

\section{Results}

Swimming exercise improves cardiac structure and function in aged mice. Swimming exercise attenuated the irregularly arranged cardiomyocytes that are caused by aging (Fig. 1A). A decrease in cardiac function is a hallmark of aging. The left ventricular ejection fraction (LVEF) and left ventricular fraction shortening (LVFS) were measured using echocardiography to determine the cardiac function of mice in the young, aged and aged + exercise groups (Fig. 1B). A significantly 
lower LVEF and LVFS were observed in aged mice compared with young mice (Fig. 1C and D). Swimming exercise significantly improved the heart function of aged mice (Fig. 1B-D). Additionally, the levels of several oxidative stress indicators, including SOD, MDA and ROS, were measured. Lower SOD activity was detected in hearts from the aged group compared with the young group, and swimming exercise significantly increased SOD activity (Fig. 1E). In addition, swimming exercise ameliorated age-induced alterations in the levels of MDA (Fig. 1F) and ROS (Fig. 1G). Based on these results, swimming exercise was found to exert beneficial effects on the heart that were found to be associated with an attenuation of oxidative stress in aged mice.

Swimming exercise inhibits ER stress in the hearts of aged mice. ER stress and oxidative stress are closely related processes (18). Therefore, experiments were conducted to examine the levels of ER stress-related proteins. ER stress was induced in the aged hearts, as indicated by the increased level of p-PERK (Fig. 2A). Furthermore, the levels of GRP78 and CHOP were also increased (Fig. 2B). The increased levels of p-PERK, GRP78 and CHOP were all suppressed by swimming exercise. Therefore, swimming exercise was found to inhibit ER stress in aged mice.

Swimming exercise reverses the downregulation of cGMP-PKG signaling in the hearts of aged mice. The effect of swimming exercise on myocardial cGMP-PKG signaling was evaluated to investigate the cardioprotective mechanism of swimming exercise in aged hearts. Significantly lower PKG expression was observed in aged hearts compared with young hearts, and this effect was significantly reversed by swimming exercise (Fig. 3A). cGMP levels in the myocardium were higher in aged mice subjected to swimming exercise than in the aged group (Fig. 3B). Furthermore, higher levels of PDE5 were detected in aged hearts compared with young hearts, and swimming exercise reduced the increase of PDE5 levels in aged hearts (Fig. 3C). Based on these results, swimming exercise was found to reverse the downregulation of cGMP-PKG signaling in aged hearts by increasing PKG and cGMP levels and reducing PDE5 levels.

Effects of the cGMP signaling pathway on cardiac function, ROS production and ER stress. To determine whether cGMP-PKG signaling regulated the anti-ER stress effect of swimming exercise in aged hearts, the aged group were treated with sildenafil, a specific PDE5 inhibitor, and the aged + exercise group were treated with TM, an ER stress inducer. The heart function in all groups was observed by echocardiography (Fig. 4A). The LVEF and LVFS values were slightly increased in the hearts of aged mice treated with sildenafil or swimming exercise compared with aged hearts (Fig. 4B and C). Additionally, sildenafil and swimming exercise significantly reduced the levels of ROS in the aged hearts (Fig. 4D). Exposure to the ER stress inducer TM, which increased the expression levels of GRP78 and CHOP proteins to mimic ER stress-induced injuries, abolished the beneficial effects of swimming exercise on cardiac function in aged mice and increased ROS production compared with swimming exercise in aged mice (Fig. 4A-D). Sildenafil decreased

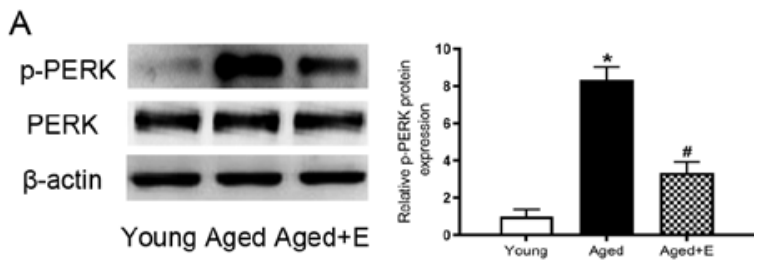

B
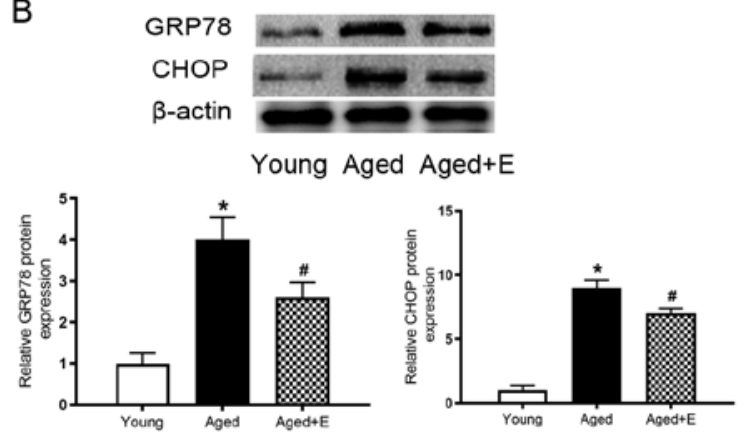

Figure 2. Swimming exercise decreases the levels of endoplasmic reticulum stress-related proteins in aged hearts. (A) Levels of p-PERK in the hearts of mice from each group. Proteins levels were normalized to PERK. (B) Levels of GRP78 and CHOP. Proteins levels were normalized to $\beta$-actin. ${ }^{*} \mathrm{P}<0.05$ vs. young mice; ${ }^{\#} \mathrm{P}<0.05$ vs. aged mice. Data are presented as the mean \pm SEM from independent experiments performed in triplicate. PERK, PKR-like ER kinase; GRP78, glucose-regulated protein 78; CHOP, C/EBP homologous protein; p-, phosphorylated; E, exercise.

the levels of the ER stress-related proteins GRP78 and CHOP (Fig. 4E), increased levels of PKG and reduced the level of PDE5 (Fig. 4F), while reducing ROS production (Fig. 4A-D). The present data suggested that the activation of cGMP-PKG signaling can mimic the protective effect of exercise in aged mice. TM did not affect PKG and PDE5 expression (Fig. 4F). However, TM increased ROS production and reduced cardiac function in aged mice after exercise (Fig. 4A-D). Collectively, the present data suggested that activation of ER stress abolished the protective effect of exercise in aged mice. In summary, swimming exercise exerted its cardioprotective effect by ameliorating ER stress through the cGMP-PKG signaling pathway.

\section{Discussion}

The present study identified that swimming exercise significantly attenuated the negative effects of aging on cardiac structure and myocardial function by suppressing the oxidative stress and ER stress responses. In addition, it was found that the cGMP-PKG cascade may serve an important role in the exercise-induced decrease in ER stress in aged hearts. The present results not only provide additional evidence supporting the cardioprotective effects of swimming exercise on reducing cardiac injuries in aged mice, but also emphasize the importance of the ER stress-dependent cGMP-PKG cascade in the protective effects of swimming exercise on the heart.

Cardiac aging alters cardiac filling function, compliance and ventricular pump capacity, thus contributing to a decrease in cardiac function (19). In the present study, the cardiac structure and function were found to be impaired in aged mice. The aging-induced deterioration of the cardiac structure and 
A

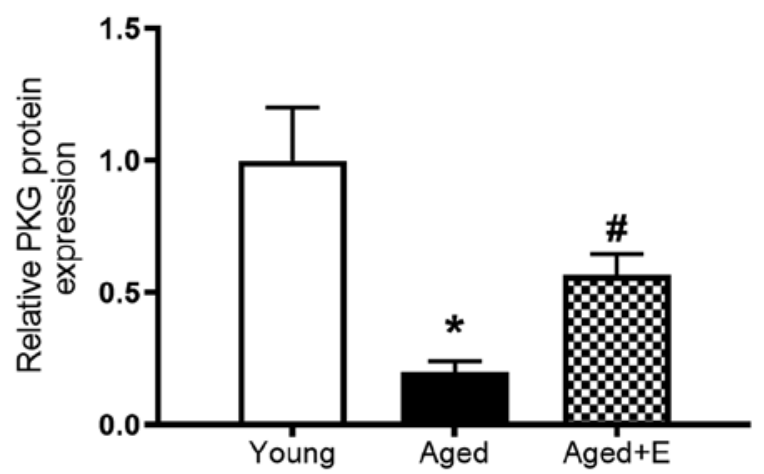

B

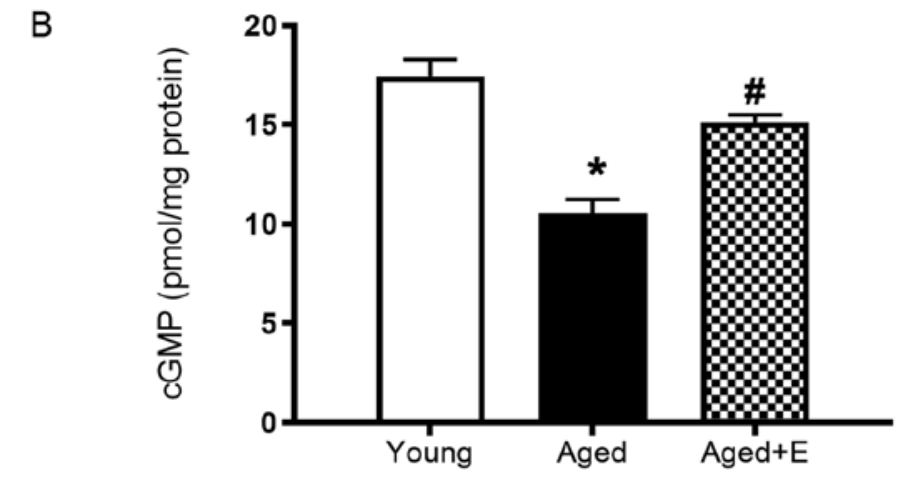

C
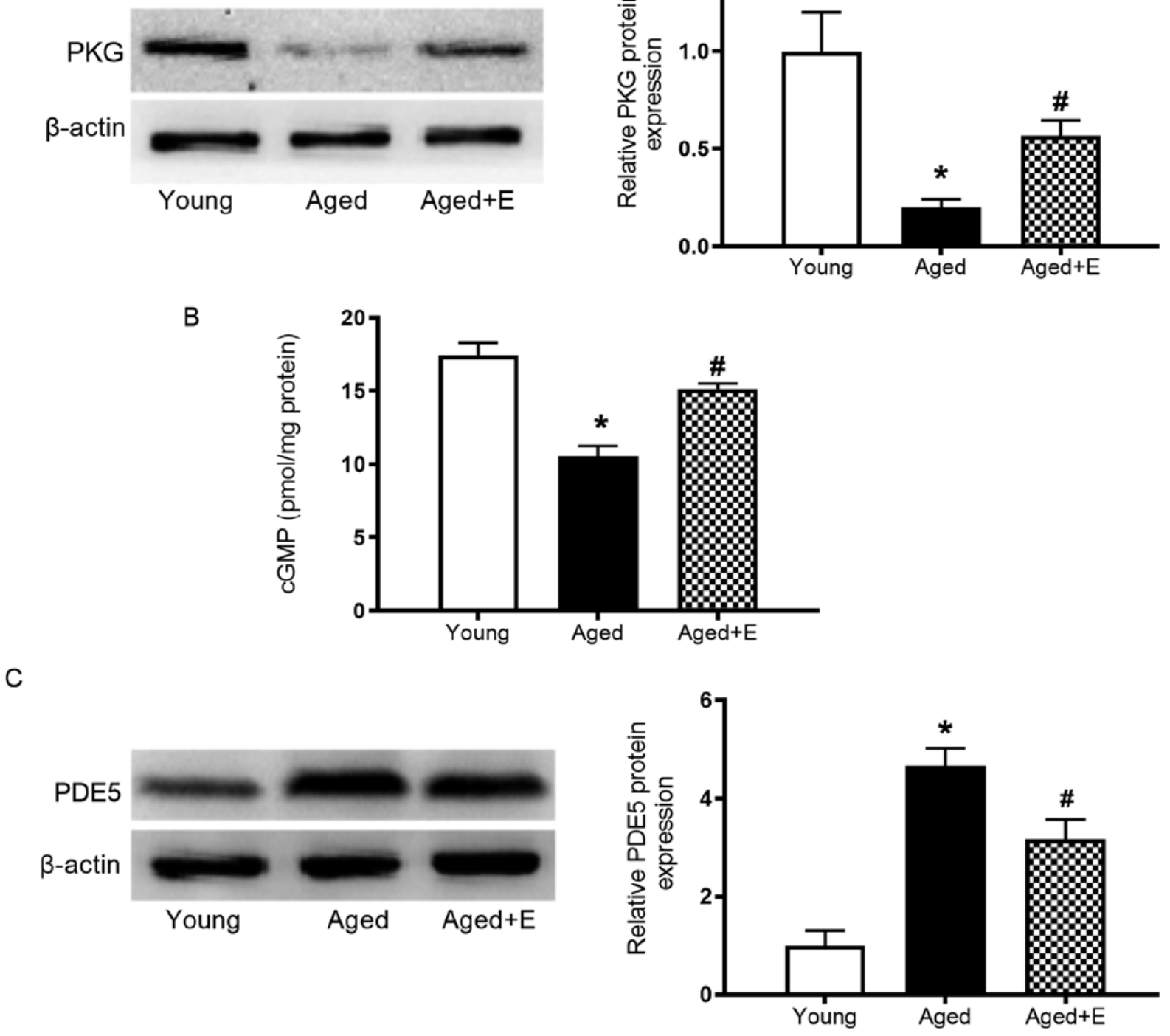

Figure 3. Swimming exercise increases the myocardial levels of cGMP and PKG, and decreases the level of PDE5 in aged mice. (A) PKG levels in the hearts of mice from each group. (B) Myocardial cGMP contents in mice. (C) PDE5 levels in the hearts of mice from each group. "P<0.05 vs. young mice; ${ }^{*} \mathrm{P}<0.05$ vs. aged mice. Data are presented as the mean \pm SEM from three independent experiments. cGMP, cyclic GMP; PKG, protein kinase G; PDE5, cGMP-specific phosphodiesterase type 5; E, exercise.

decreased cardiac function were attenuated in mice performing swimming exercise. Therefore, swimming exercise improved aging-induced impairments in cardiac performance. Specific increases in oxidative stress have been reported to represent potential factors that determine the induction and maintenance of cellular senescence, and the aging process $(8,20,21)$. An imbalance in ROS generation triggers oxidative stress, and ROS play an important role in many physiological and pathological processes (22). Additionally, SOD and MDA are involved in oxidative stress defense (23). Regular physical exercise delays the accumulation of ROS-mediated cell damage by improving antioxidative protective mechanisms in the myocardium (24). Exercise has been reported to increase SOD mRNA expression (25). As shown in the present study, aging decreased SOD activity and increased MDA levels. However, swimming exercise exerted beneficial effects by increasing the activity of the antioxidant enzyme SOD, and by decreasing MDA and ROS production.
Accumulating evidence demonstrated that oxidative stress and the ER stress are associated events $(17,18)$. The ER-localized transmembrane kinase PERK is a major transducer of ER stress (26). Normally, PERK is maintained in an inactive, monomeric state by binding to GRP78. When this binding is disrupted, PERK homodimerizes, undergoes autophosphorylation, becomes active and initiates downstream signaling (27). Protein accumulation in the ER results in ER stress and ultimately activates apoptosis. This pathway involves the upregulation of CHOP (28). CHOP levels are elevated in the liver in response to aging (29). Consistent with this previous study, increased levels of CHOP were observed in aged hearts in the present study. Swimming exercise reduced CHOP activity. Moreover, in the present study, swimming exercise inhibited the increase in GRP78 expression in aged hearts. The present results suggested that swimming exercise protected the hearts of aged mice from oxidative stimulation and ER stress. 
A
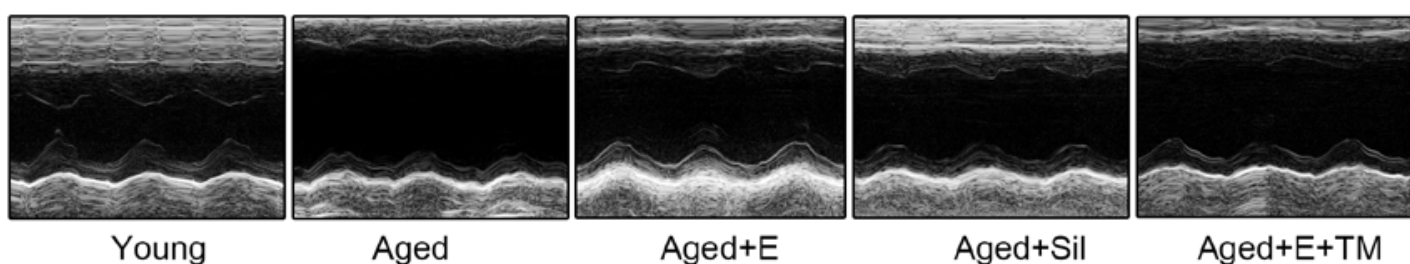

B
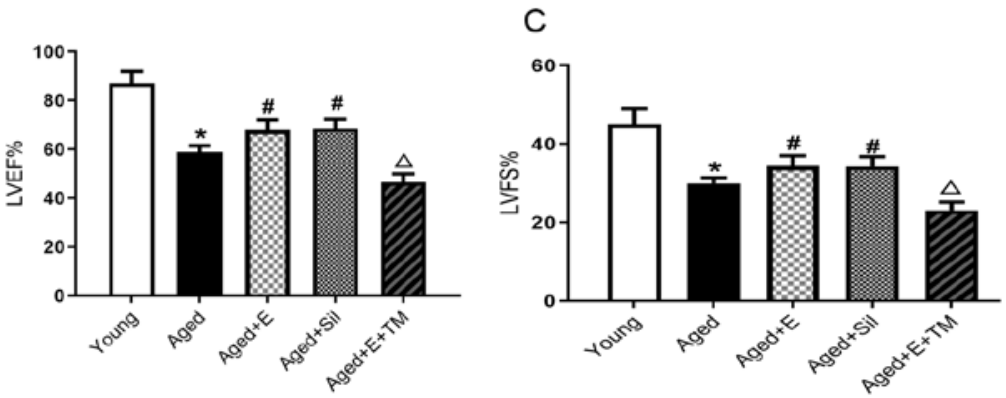

Aged+Sil

Aged+E+TM

E
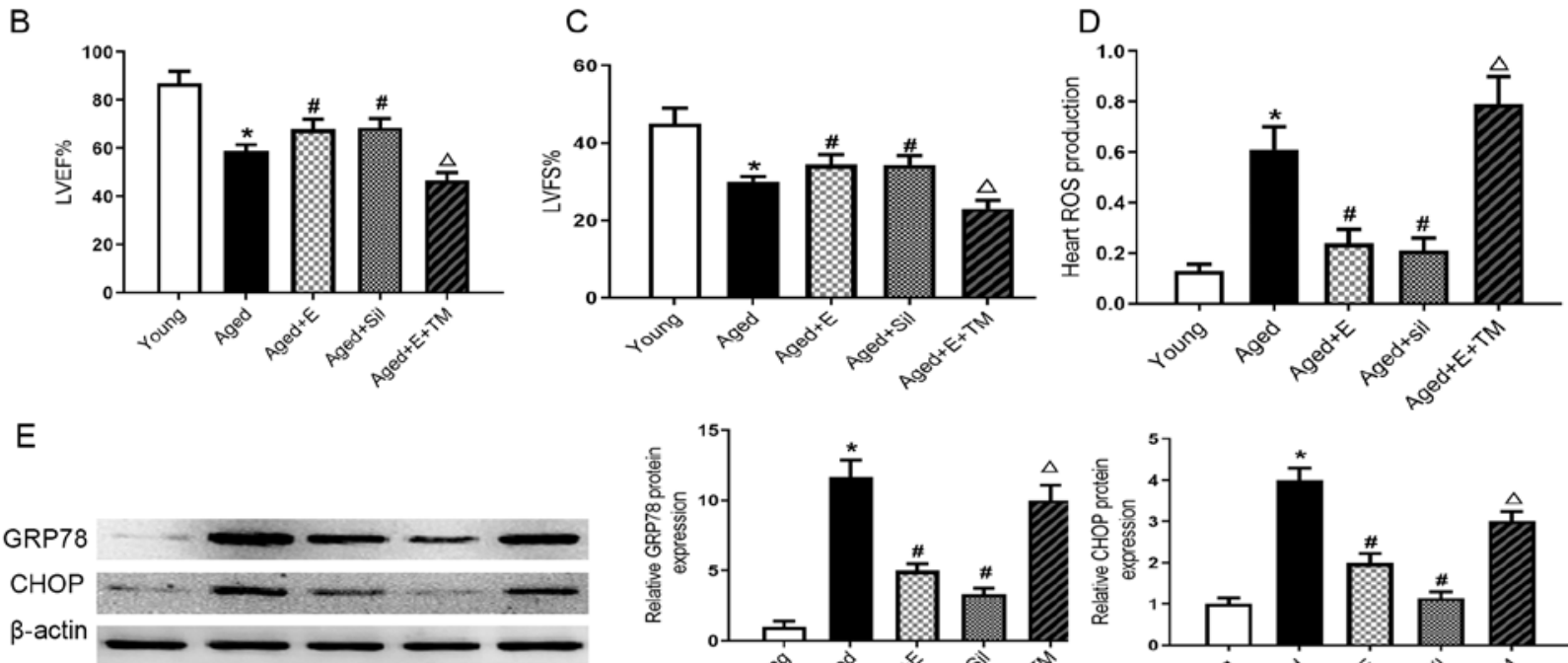

Young Aged Aged+E Aged+Sil Aged+E+TM
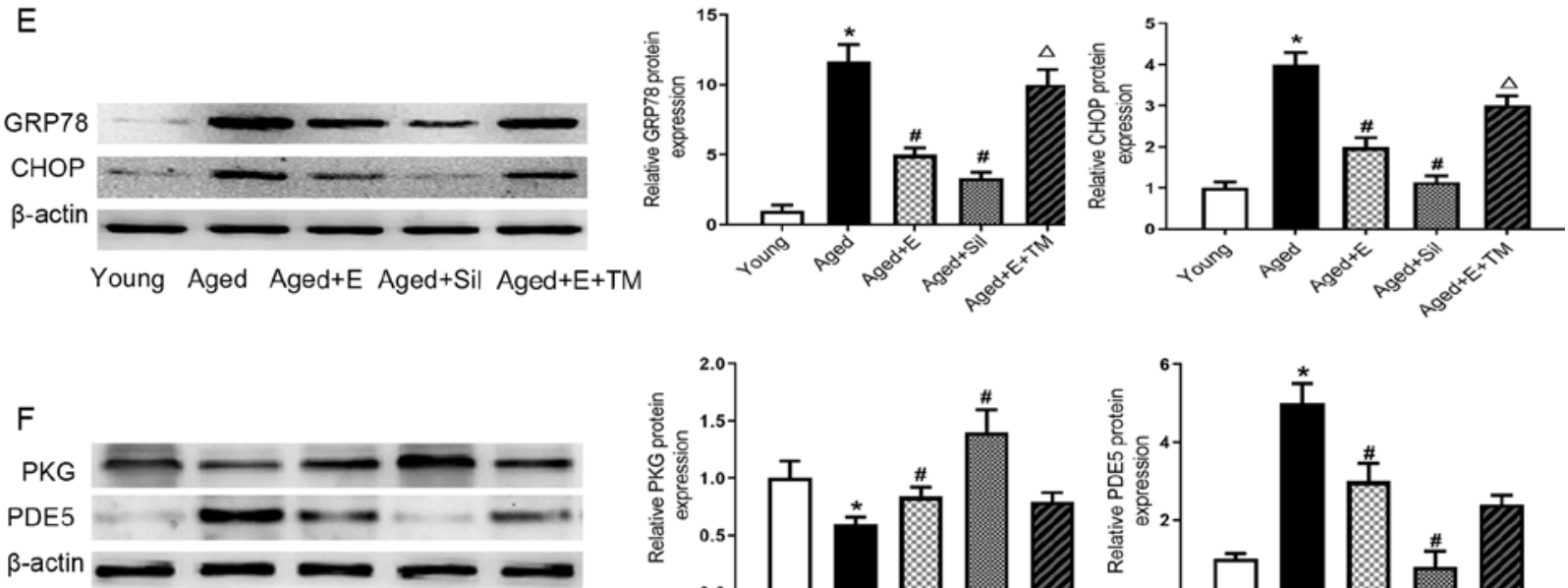

Young Aged Aged+E Aged+Sil Aged+E+TM
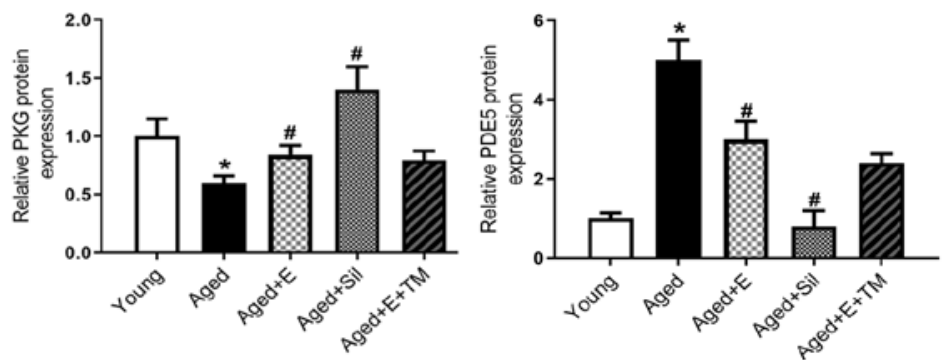

Figure 4. Activation of cGMP inhibits ROS production and endoplasmic reticulum stress. (A) Echocardiography of hearts from mice in each group. (B) LVEF and (C) LVFS of hearts from mice in each group. (D) ROS production. (E) Levels of GRP78 and CHOP. (F) Levels of PKG and PDE5. Proteins were normalized to $\beta$-actin. ${ }^{*} \mathrm{P}<0.05$ vs. young mice; ${ }^{\#} \mathrm{P}<0.05$ vs. aged mice; ${ }^{\Delta} \mathrm{P}<0.05$ vs. aged mice $+\mathrm{E}$. Data are presented as the mean \pm SEM of three independent experiments. LVEF, left ventricle ejection fraction; LVFS, left ventricle fractional shortening; ROS, reactive oxygen species; GRP78, glucose-regulated protein 78; CHOP, C/EBP homologous protein; PKG, protein kinase G; PDE5, cGMP-specific phosphodiesterase type 5; E, exercise; Sil, sildenafil; TM, tunicamycin.

The primary mediator of cGMP signaling is PKG, which in turn phosphorylates multiple intracellular proteins in the cardiovascular system (30). Both cGMP and its downstream effector, the PKG signaling pathway, are associated with markedly reduced infarct sizes (15) and regulate cardiac function (13). Previously, aging has been reported to be associated with the downregulation of cGMP-PKG signaling in vascular smooth muscle cells (31) and postmenopausal women (32). The previous studies provided evidence supporting the relationship between aging and the increased risk of cardiovascular disease. The levels of cGMP and PKG activity were decreased in aged hearts in the present study, indicating that aging impaired myocardial cGMP-PKG signaling. Consistent with the present results, previous studies reported a significant downregulation of the cGMP-PKG axis in diabetic animals (33) and in response to myocardial ischemia/reperfusion injury (14). Therefore, decreased cGMP-PKG signaling may be associated with aggravated damage induced by a myocardial insult.
The present study suggested that the reduced levels of cGMP and PKG activity in the heart were ameliorated in aged mice following swimming exercise. Taken together, the findings of the present study indicated that the cGMP-PKG signaling pathway may be involved in the protective effects of swimming exercise against the aging-induced decline in cardiac function.

According to previous in vitro and in vivo studies, cardiac cGMP levels are increased following inhibition of the cGMP-degrading enzyme PDE5 (33-35). PDE5 is expressed at low levels in the heart under normal physiological conditions (36). PDE5 expression is upregulated in myocardial samples from patients with different heart diseases (37-39). Theoretically, the blockade of the pathological effects of PDE5 should exert cytoprotective effects against different cardiovascular diseases. Similarly, PDE5 inhibitors are useful treatments that improve cGMP signaling to reduce cardiomyocyte stiffness $(34,35)$. In the present study, PDE5 expression 
was increased in aged hearts. Furthermore, PDE5 expression was decreased in aged mice after swimming exercise or sildenafil treatment. The results of the present study further support the hypothesis that the effects of swimming exercise on aged hearts are potentiated via the activation of the cGMP-PKG signaling pathway.

ER stress is increased in response to aging and cardiovascular disease (40). The cGMP-PKG signaling pathway has been reported to contribute to cardioprotective mechanisms (14). Gong et al (41) reported a significant correlation between increased PDE5 expression and the activation of ER stress in failing hearts. However, few studies have examined the effects of the cGMP-PKG pathway on ER stress in aged hearts. In the present study, treatment with the PDE5 inhibitor sildenafil or swimming exercise attenuated myocardial injury and levels of ER stress-related proteins. A specific inducer of ER stress, TM, was used to determine whether the activity of the cGMP signaling pathway was suppressed by ER stress. TM abolished the beneficial effects of swimming exercise on the aged hearts. Based on the results from the present study, age-induced cardiac dysfunction is associated with enhanced oxidative stress and ER stress. By contrast, swimming exercise improved cardiac function by inhibiting the oxidative stress and ER stress responses, which were mediated through activation of the cGMP-PKG signaling pathway.

Collectively, the present study suggested that swimming exercise improved myocardial function and exerted beneficial effects on aged hearts. The underlying mechanism may be explained by a swimming exercise-mediated increase in cGMP-PKG signaling, causing an increase in the antioxidant response and reductions in ER stress. The present study provided new insights into the myocardial effects of physical activity, which may facilitate the identification of novel therapeutic regimens for age-related cardiovascular diseases.

\section{Acknowledgements}

Not applicable.

\section{Funding}

The present study was supported by grants from the Natural Science Foundation of China (grant nos. 81870172, 81670365, 81470438, 81601306 and 81671648) and the Key Problems of Social Development Science and Technology of Shaanxi Province (grant nos. 2018SF-129, 2018ZDXM-SF-068 and 2018SF-114).

\section{Availability of data and materials}

The datasets used and/or analyzed during the present study are available from the corresponding author on reasonable request.

\section{Authors' contributions}

PC, XMZ, MYZ, XLZ, MZZ and JY conceived and designed the research. PC, XMZ, MYZ, GL, LH, HZ, JW, XW, KW, JZ, MR, BC, XXZ, XLZ, MZZ and JY performed experiments. PC, XMZ, MYZ, GL, LH, HZ, JW, XW, MZZ, JY and KW analyzed data. PC, XMZ, MYZ, JZ, BC, XXZ, XLZ, MZZ,
JY and MR drafted the manuscript. PC, XMZ, MYZ and MZZ and JY edited and revised the manuscript. All authors read and approved the final version of the manuscript.

\section{Ethics approval and consent to participate}

All animal experimental procedures and protocols were approved by The Ethics Committee of The Fourth Military Medical University (Xi'an, China).

\section{Patient consent for publication}

Not applicable.

\section{Competing interests}

The authors declare that they have no competing interests.

\section{References}

1. Yang X, Sreejayan N and Ren J: Views from within and beyond: Narratives of cardiac contractile dysfunction under senescence. Endocrine 26: 127-137, 2005

2. Wu J, Xia S, Kalionis B, Wan W and Sun T: The role of oxidative stress and inflammation in cardiovascular aging. Biomed Res Int 2014: 615312, 2014.

3. Lakatta EG and Levy D: Arterial and cardiac aging: Major shareholders in cardiovascular disease enterprises: Part II: The aging heart in health: Links to heart disease. Circulation 107: 346-354, 2003.

4. Ozturk N, Olgar Y, Er H, Kucuk M and Ozdemir S: Swimming exercise reverses aging-related contractile abnormalities of female heart by improving structural alterations. Cardiol J 24: 85-93, 2017.

5. Bachschmid MM, Schildknecht S, Matsui R, Zee R, Haeussler D, Cohen RA, Pimental D and Loo B: Vascular aging: Chronic oxidative stress and impairment of redox signaling-consequences for vascular homeostasis and disease. Ann Med 45: 17-36, 2013.

6. Belaya I, Suwa M, Chen T, Giniatullin R, Kanninen KM, Atalay $\mathrm{M}$ and Kumagai S: Long-term exercise protects against cellular stresses in aged mice. Oxid Med Cell Longev 2018: 2894247, 2018.

7. Kim SR and Lee YC: Endoplasmic reticulum stress and the related signaling networks in severe asthma. Allergy Asthma Immunol Res 7: 106-117, 2015.

8. Lesnefsky EJ, Chen Q and Hoppel CL: Mitochondrial metabolism in aging heart. Circ Res 118: 1593-1611, 2016.

9. da Luz G, Frederico MJ, da Silva S, Vitto MF, Cesconetto PA, de Pinho RA, Pauli JR, Silva AS, Cintra DE, Ropelle ER and De Souza CT: Endurance exercise training ameliorates insulin resistance and reticulum stress in adipose and hepatic tissue in obese rats. Eur J Appl Physiol 111: 2015-2023, 2011.

10. Wang Y, Wang S, Wier WG, Zhang Q, Jiang H, Li Q, Chen S, Tian Z, Li Y, Yu X, et al: Exercise improves the dilatation function of mesenteric arteries in postmyocardial infarction rats via a PI3K/Akt/eNOS pathway-mediated mechanism. Am J Physiol Heart Circ Physiol 299: H2097-H2106, 2010.

11. Zhang QJ, Li QX, Zhang HF, Zhang KR, Guo WY, Wang HC, Zhou Z, Cheng HP, Ren J and Gao F: Swim training sensitizes myocardial response to insulin: Role of Akt-dependent eNOS activation. Cardiovasc Res 75: 369-380, 2007.

12. Puzzo D, Loreto C, Giunta S, Musumeci G, Frasca G, Podda MV, Arancio $\mathrm{O}$ and Palmeri A: Effect of phosphodiesterase-5 inhibition on apoptosis and beta amyloid load in aged mice. Neurobiol Aging 35: 520-531, 2014.

13. Takimoto E, Champion HC, Li M, Belardi D, Ren S, Rodriguez ER, Bedja D, Gabrielson KL, Wang Y and Kass DA: Chronic inhibition of cyclic GMP phosphodiesterase 5A prevents and reverses cardiac hypertrophy. Nat Med 11: 214-222, 2005.

14. Inserte $J$ and Garcia-Dorado D: The cGMP/PKG pathway as a common mediator of cardioprotection: Translatability and mechanism. Br J Pharmacol 172: 1996-2009, 2015. 
15. Yu LM, Di WC, Dong X, Li Z, Zhang Y, Xue XD, Xu YL, Zhang J, Xiao X, Han JS, et al: Melatonin protects diabetic heart against ischemia-reperfusion injury, role of membrane receptor-dependent cGMP-PKG activation. Biochim Biophys Acta Mol Basis Dis 1864: 563-578, 2018.

16. Prola A, Pires Da Silva J, Guilbert A, Lecru L, Piquereau J, Ribeiro M, Mateo P, Gressette M, Fortin D, Boursier C, et al: SIRT1 protects the heart from ER stress-induced cell death through eIF2 $\alpha$ deacetylation. Cell Death Differ 24: 343-356, 2017.

17. Chang P, Zhang M, Zhang X, Li G, Hu H, Wu J, Wang X, Yang Z, Zhang J, Chen W, et al: B-type natriuretic peptide attenuates endoplasmic reticulum stress in $\mathrm{H} 9 \mathrm{c} 2$ cardiomyocytes underwent hypoxia/reoxygenation injury under high glucose/high fat conditions. Peptides 111: 103-111, 2019.

18. Malhotra JD and Kaufman RJ: Endoplasmic reticulum stress and oxidative stress: A vicious cycle or a double-edged sword? Antioxid Redox Signal 9: 2277-2293, 2007.

19. Zhang Y, Wang C, Zhou J, Sun A, Hueckstaedt LK, Ge J and Ren J: Complex inhibition of autophagy by mitochondrial aldehyde dehydrogenase shortens lifespan and exacerbates cardiac aging. Biochim Biophys Acta Mol Basis Dis 1863: 1919-1932, 2017.

20. Gambino V, De Michele G, Venezia O, Migliaccio P, Dall'Olio V, Bernard L, Minardi SP, Della Fazia MA, Bartoli D, Servillo G, et al: Oxidative stress activates a specific p53 transcriptional response that regulates cellular senescence and aging. Aging Cell 12: 435-445, 2013.

21. Vigneron A and Vousden KH: p53, ROS and senescence in the control of aging. Aging (Albany NY) 2: 471-474, 2010.

22. Sikka SC and Hellstrom WJ: Role of oxidative stress and antioxidants in Peyronie's disease. Int J Impot Res 14: 353-360, 2002.

23. Amin MM, Rafiei N, Poursafa P, Ebrahimpour K, Mozafarian N, Shoshtari-Yeganeh B, Hashemi M and Kelishadi R: Association of benzene exposure with insulin resistance, SOD, and MDA as markers of oxidative stress in children and adolescents. Environ Sci Pollut Res Int 25: 34046-34052, 2018.

24. Golbidi S and Laher I: Exercise and the cardiovascular system. Cardiol Res Pract 2012: 210852, 2012.

25. Zhao H, Liu J, Pan S, Sun Y, Li Q, Li F, Ma L and Guo Q: SOD mRNA and MDA expression in rectus femoris muscle of rats with different eccentric exercise programs and time points. PLoS One 8: e73634, 2013.

26. Cui W, Li J, Ron D and Sha B: The structure of the PERK kinase domain suggests the mechanism for its activation. Acta Crystallogr D Biol Crystallogr 67: 423-428, 2011.

27. Sanderson TH, Deogracias MP, Nangia KK, Wang J, Krause GS and Kumar R: PKR-like endoplasmic reticulum kinase (PERK) activation following brain ischemia is independent of unfolded nascent proteins. Neuroscience 169: 1307-1314, 2010.

28. Delbrel E, Soumare A, Naguez A, Label R, Bernard O, Bruhat A, Fafournoux P, Tremblais G, Marchant D, Gille T, et al: HIF-1 $\alpha$ triggers ER stress and CHOP-mediated apoptosis in alveolar epithelial cells, a key event in pulmonary fibrosis. Sci Rep 8: 17939, 2018.

29. Ikeyama S, Wang XT, Li J, Podlutsky A, Martindale JL, Kokkonen G, van Huizen R, Gorospe M and Holbrook NJ: Expression of the pro-apoptotic gene gadd153/chop is elevated in liver with aging and sensitizes cells to oxidant injury. J Biol Chem 278: 16726-16731, 2003
30. Breivik L, Jensen A, Guvåg S, Aarnes EK, Aspevik A, Helgeland E, Hovland S, Brattelid T and Jonassen AK: B-type natriuretic peptide expression and cardioprotection is regulated by Akt dependent signaling at early reperfusion. Peptides 66: 43-50, 2015.

31. Lin CS, Liu X, Tu R, Chow S and Lue TF: Age-related decrease of protein kinase $\mathrm{G}$ activation in vascular smooth muscle cells. Biochem Biophys Res Commun 287: 244-248, 2001.

32. Cui R, Iso H, Yamagishi K, Ohira T, Tanigawa T, Kitamura A, Kiyama $M$, Imano $H$, Konishi $M$ and Shimamoto $T$ : Relationship of urinary cGMP excretion with aging and menopausal status in a general population. J Atheroscler Thromb 16: 457-462, 2009.

33. Mátyás C, Németh BT, Oláh A, Török M, Ruppert M, Kellermayer D, Barta BA, Szabó G, Kökény G, Horváth EM, et al: Prevention of the development of heart failure with preserved ejection fraction by the phosphodiesterase-5A inhibitor vardenafil in rats with type 2 diabetes. Eur J Heart Fail 19: 326-336, 2017.

34. Wang L, Chopp M, Szalad A, Liu Z, Bolz M, Alvarez FM, Lu M, Zhang L, Cui Y, Zhang RL and Zhang ZG: Phosphodiesterase-5 is a therapeutic target for peripheral neuropathy in diabetic mice. Neuroscience 193: 399-410, 2011.

35. Zhang $M$ and Kass DA: Phosphodiesterases and cardiac cGMP: Evolving roles and controversies. Trends Pharmacol Sci 32: 360-365, 2011

36. Cheitlin MD, Hutter AM Jr, Brindis RG, Ganz P, Kaul S, Russell RO Jr and Zusman RM: ACC/AHA expert consensus document. Use of sildenafil (Viagra) in patients with cardiovascular disease. American College of Cardiology/American Heart Association. J Am Coll Cardiol 33: 273-282, 1999.

37. Rashid M, Kotwani A and Fahim M: Long-acting phosphodiesterase 5 inhibitor, tadalafil, and superoxide dismutase mimetic, tempol, protect against acute hypoxia-induced pulmonary hypertension in rats. Hum Exp Toxicol 31: 626-636, 2012.

38. Lu Z, Xu X, Hu X, Lee S, Traverse JH, Zhu G, Fassett J, Tao Y, Zhang P, dos Remedios C, et al: Oxidative stress regulates left ventricular PDE5 expression in the failing heart. Circulation 121: 1474-1483, 2010

39. Kukreja RC, Salloum FN and Das A: Cyclic guanosine monophosphate signaling and phosphodiesterase-5 inhibitors in cardioprotection. J Am Coll Cardiol 59: 1921-1927, 2012.

40. Zhang C, Syed TW, Liu R and Yu J: Role of endoplasmic reticulum stress, autophagy, and inflammation in cardiovascular disease. Front Cardiovasc Med 4: 29, 2017.

41. Gong W, Duan Q, Cai Z, Chen C, Ni L, Yan M, Wang X, Cianflone K and Wang DW: Chronic inhibition of cGMP-specific phosphodiesterase 5 suppresses endoplasmic reticulum stress in heart failure. Br J Pharmacol 170: 1396-1409, 2013.

This work is licensed under a Creative Commons Attribution-NonCommercial-NoDerivatives 4.0 International (CC BY-NC-ND 4.0) License. 